\title{
Analysis on the Commutation Process of the Single-phase Rectifier Circuit and its Application
}

\author{
Kuo Zhao ${ }^{1,}$, Dong $\mathrm{Xu}^{2}$ \\ ${ }^{1}$ Chongqing College of Electronic Engineering ,Chongqing China 401331 \\ ${ }^{2}$ Chongqing College of Electronic Engineering ,Chongqing China 401331 \\ a zhkyes@163.com
}

Keywords: Single phase; Rectifier circuit; Commutation; Application

\begin{abstract}
With the maturity and popularization of the technology of power electronic transformation, the power drag, rectifier circuit is used in great quantities in the application fields such as power system, which is including diode uncontrolled rectifier circuit, voltage type PWM rectifier circuit. To stabilize the DC voltage loop, the high-power power electronic converter requires the use of high capacity values of the electrolytic capacitor. Therefore in the rectifier circuit electric surge current limiting measures must be taken, otherwise it will cause the movement, the air switch fuse burn out, the problem such as line loss is too large, also due to the presence of stray inductance level inverter circuit over-voltage breakdown after making. This paper will focus on the single-phase rectifier circuit fazed rectifier circuit and inverter structure, properties, and other elements and single phase rectifier circuit selection factors in the process of application.
\end{abstract}

\section{Introduction}

With characteristics of large inductance rectifier circuit has been widely used in power transmission, medium frequency induction heating, stable power supply, etc. Due to the inductance load, rectifier circuit of input current waveform approximation for the square wave. In order to reduce the negative impact of the rectifier circuit for power grid and the short circuit current limiting, often in the input end of the rectifier circuit with the rectifier transformer or communication into line reactor, so that the rectifier circuit commutation overlap Angle during commutation on phenomenon. In this paper, the existing transformer leakage resistance of the commutation process of the cases of single-phase rectifier circuit and overlap Angle, change the quantitative calculation of pressure drop are analyzed in detail, and through the computer simulation and experimental results are verified..

\section{The single phase rectifier circuit}

Usually, different load properties of the rectifier circuit are connected, to produce their own different characteristics. Through the differences, single-phase rectifier circuit can be further divided into three categories, followed by single-phase half-wave controlled rectifier circuit, single-phase full wave controlled rectifier circuit and single phase bridge rectifier circuit.

The door single-phase half-wave controlled rectifier circuit. Single-phase half-wave controlled rectifier circuit when the alternating current in the input for the single phase, at the same time, also will produce a dc output voltage waveform, and only in the ac input is half weeks. Single-phase half-wave controlled rectifier circuit has its distinctive characteristics, namely the circuit is simple, and the output pulse is opposite bigger. Due to this type of controlled rectifier circuit in the communication loop as stated earlier in the types of dc component, and thus easy to cause converter transformer iron core saturation, which reduces the efficiency of the equipment.

The single-phase full wave controlled rectifier circuit. Single-phase full wave controlled rectifier circuit is also a type of single-phase rectifier circuit; its structure is relatively simple. In addition, in the trigger circuit requirements, the requirements of the trigger are relatively low. This type of rectifier circuit output dc voltage pulse is opposite bigger, than other types of circuit, 
single-phase full wave controlled rectifier circuit are more likely to lead to power grid load imbalance. In view of the above characteristics, single phase rectifier circuit usually in less than 4 $\mathrm{kW}$ power small or medium or small and medium capacity of the use of electrical equipment.

The single-phase bridge type all control rectifier circuit. Single-phase bridge type all control rectifier circuit is very widely used class of rectifier circuit, the rectifier circuit structure is simple, just an inductance, four thyristor and a resistor, so the manufacturing cost is low. Single-phase bridge type all control the positive half cycle of the rectifier circuit in ac power and negative half cycle will have rectifier output current flows through the load, in addition, compared with half-wave rectifier circuit, the rectifier voltage waveform of the rectifier circuit pulse more often. In the rectifier circuit of transformer primary winding, its special performance determines the transformer dc magnetization does not exist in the problem, and transformer efficiency greatly increased. Thanks to the positive half cycle and negative half cycle of the power flow to reverse flow, and the waveform symmetry of the two factors, there is no dc component, and therefore does not exist in the transformer dc magnetization problem, thus greatly improving the efficiency of transformer winding. Single phase bridge type all control rectifier circuit and single-phase half-wave controlled rectifier circuit is both similarities and there are obvious different. First of all, both within $0^{\circ}-180^{\circ}$ mobile, the mobile range is the same, but the single-phase bridge type all control the output voltage of the rectifier circuit average higher, the half-wave rectifier circuit for its only $1 / 2$, so if it is under the same load power of, through the single phase bridge rectifier circuit of thyristor will half-wave rectifier circuit can reduce the average current $1 / 2$, and power factor.

a)

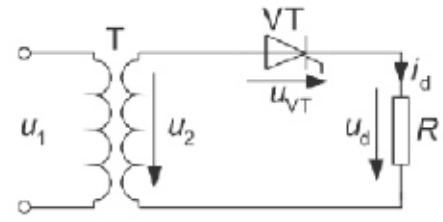

b)

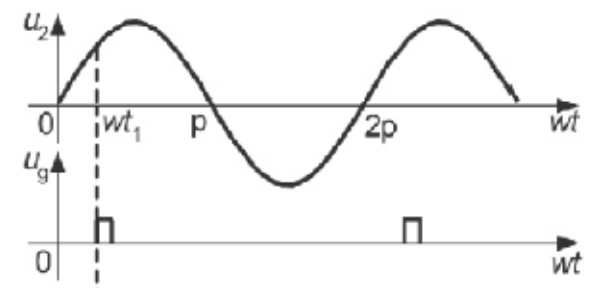

d)

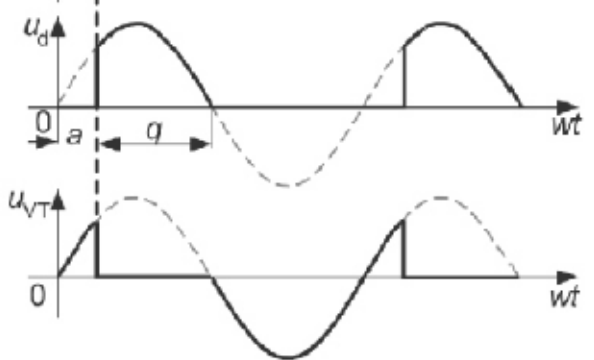

Fig. 1 Single-phase half-wave controlled rectifier circuit

\section{The active controlled rectifier and inverter}

Inverter, the direct current into alternating current (AC), the rectifying is the inverse process; Inverter circuit, the DC to AC inverter circuit. Active inverter circuit, and the ac power grid connection, applied to dc reversible speed control system, ac wound rotor asynchronous motor cascade speed regulation and high voltage direct current transmission, etc. For controlled rectifier circuit, can work in a certain condition of active inverter state, its circuit form has not changed, just shift circuit working conditions. Active inverter can also serve as a working state of it. Inverter, once the commutation failure, make the average output voltage of the converter comes at a time, and DC consequent series, forming a lot of short circuit current. The controlled rectifier ac side joint has a power frequency ac power, the average output of the dc voltage can be controlled continuously from is the maximum value to a maximum of negative, but the direction of the 
controlled rectifier dc current does not change. Among them, the first quadrant of the average dc voltage and dc current are positive, the rectifier operation, energy from the ac input to the dc side, the circuit is the inverter. Thyristor at work may withstand the most-positive, reverse voltage of the power supply voltage peak. The working principle of resistive load: in practice, some is basically a resistive load, such as resistance furnace, electrolysis and electroplating, etc. Resistive load is proportional to the voltage and current, is the feature of waveform and phase, with same currents can be mutated. The working principle of inductive load: inductive load is usually excitation coil of the motor and load series reactor, etc. When change current through the inductor, the induced potential on both ends of the inductance, induction electric potential has prevent effect to the change of the load current, load current not mutation. When the current increases, the inductance energy absorption energy storage, the inductance of the induction electric potential to prevent current increase, Current, small inductance release energy, induction electric potential to stop the decrease of the current, output voltage and current phase difference.

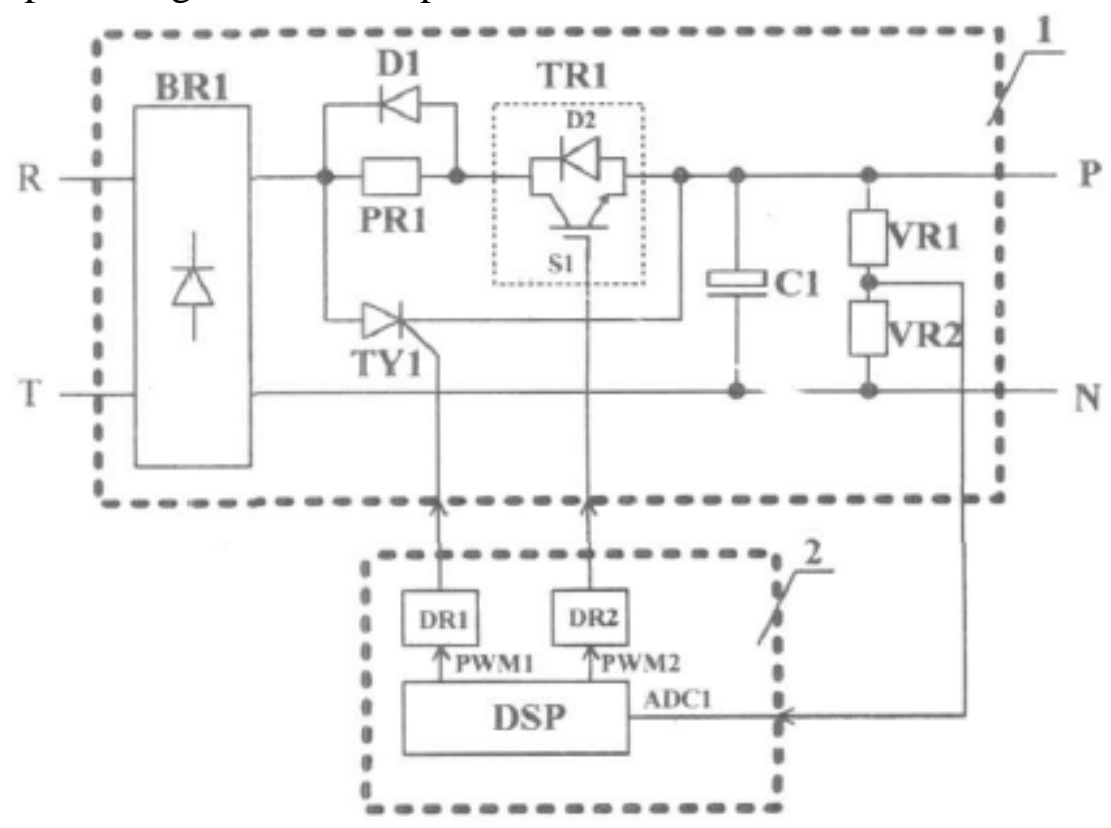

Fig. 2 Single-phase rectifier circuits on the control of the electric circuit

\section{The rectifier circuit of harmonic and power factor}

Rectifier circuit rectifier is pulsating dc voltage, output voltage rectifier output current waveform of ten large inductive loads is flat, but for ten resistances, low inductance load is still fluctuating. At the same time, the ac power current waveform, the rectifier transformer is secondary current waveform distortion, non sinusoidal. In the non-sinusoidal circuit, active power, apparent power, power factor and sinusoidal circuit defined in the same way. In the utility grid, usually voltage waveform distortion is small, but current waveform distortion may be large, so the voltage waveform is sine wave, it is of practical significance for the current waveform is the sine wave. Usually pulse wave number, the more output in dc side is smooth, the closer the sine wave ac current. In order to increase the number of pulse wave, can increase the communication phase number, but the general increase in the number, the more is less time for each phase of electricity, such meetings make rectifier components and vice winding rectifier transformer utilization becomes bad, make the device volume is big, the cost increase. The influence of harmonic and power factor of power grid:

Harmonic generation power electronic devices, cause harm to the utility grid, the power supply voltage and current waveform distortion. Power supply voltage and current waveforms not only affect the other users of the power grid, the will to power electronic device itself, such as synchronous voltage distortion makes the triggering Angle is not stable, lead to rectifying waveform is irregular. Increase the load and the line current, take up the power supply capacity, and make 
components produce additional loss in power grid, power factor, and lower efficiency. The harmonic load torque motor does not, for the additional harmonic loss and fever, shorten the service life of the equipment, to close communication system to produce interference. By the switching process factors such as rapidity, high voltage large power flow will produce electromagnetic interference within a certain range, the normal operation of the communication equipment. On the power supply used for power factor compensation capacitors in parallel to overheat. Because the capacitor high frequency impedance is low, it is easy to through a lot of harmonic current, caused by higher harmonic current amplification; serious harmonic overload might damage the capacitor. May produce harmonic resonance over voltage make the harmonic amplification, cable breakdown caused by accidents? Harmonic negative sequence characteristics are easy to make the relay protection and automatic device sensitive components such as false action. Reduce the precision of the measuring instrument. A large amount of harmonic and 3 times 3 times of several harmonic flows through the neutral wire can make the center line overload.

Consumption of reactive power, power electronic devices will be detrimental to the utility grid, lead to the increase of the apparent power, thereby increasing the capacity of the power supply. Make the total current is added, so that the line loss increases. Impact of reactive load can make the sharp fluctuations in the grid voltage. Ways of raising power factor mainly has: select the appropriate input voltage, in the case of control and the adjusting range is minimize control Angle of a. Increase of rectifier phase, improve the alternating current waveform, and reduce the harmonic component. Set up a compensation capacitor and filter, and using high power factor rectifier circuit, such as PWM rectifier circuit.

\section{Summary}

The rectifier circuit according to the output form can be divided into half wave and full wave rectifier; they are widely used in electronic technology. That can use various methods to discuss the problem of power output of the rectifier circuit. This paper is using the Fourier series of harmonic analysis method, the integral in direct contrast calculation and graphic analysis, three different methods, such as taking single-phase full-wave rectifier circuit as an example, discusses some problems of rectifier circuit transmission power. In the single-phase full-wave rectifier circuit, the vice side voltage transformer transmission power is equal to the load resistance on the consumption of power, and the single-phase half-wave rectifier circuit also has the similar results.

\section{References}

[1] Malinowski M, Kazmierkowski M P, Hansen S,et al. Virtual-flux-based direct power control of three-phase PWM rectifiers. IEEE Transactions on Industry Applications. 2011.

[2] Noguchi T, Tomiki H, Kondo S,et al. Direct power control of PWM converter without power-source voltage sensors. IEEE Transactions on Industry Applications. 2012.

[3] Mariusz Malinowski, Marek Jasinski, Marian P Kazmierkowski.'” Simple Direct Power Control Of Three-Phase PWM Rectifier Using Space-Vector Modulation (DPC-SVM)'”. IEEE Transactions on Industrial Electronics. 2011.

[4] A.Farhadi. Modeling , Simulation and Reduction of Conducted Electromagnetic Interference Due to a PWM Buck Type Switching Power Supply. 13th International Conference on Harmonics and Quality of Power. 2012. 